\title{
Changes in the Reflux Symptom Index After Multilevel Surgery for Obstructive Sleep Apnea
}

\author{
Su Jin $\mathrm{Kim}^{1} \cdot$ Hyo Yeol Kim² $\cdot$ Jong In Jeong ${ }^{3} \cdot$ Sang Duk Hong² $\cdot$ Seung-Kyu Chung ${ }^{2} \cdot$ Hun-Jong Dhong ${ }^{2}$ \\ ${ }^{1}$ Department of Otorhinolaryngology, Eulji University Hospital, Eulji University School of Medicine, Daejeon; \\ ${ }^{2}$ Department of Otorhinolaryngology-Head and Neck Surgery, Samsung Medical Center, Sungkyunkwan University School of Medicine, Seoul; \\ ${ }^{3}$ Department of Otolaryngology, Keimyung University School of Medicine, Daegu, Korea
}

Objectives. This study evaluated whether the symptoms of laryngopharyngeal reflux (LPR) change after multilevel surgery for obstructive sleep apnea (OSA).

Methods. Patients who underwent multilevel surgery for OSA between April 2009 and September 2014 were enrolled in this study. All patients underwent preoperative polysomnography prior to surgery and were asked to complete the reflux symptom index (RSI) questionnaire before and after surgery.

Results. Of 73 enrolled patients, 24 (33\%) reported an RSI score $>13$ and were thus classified as having reflux. The mean RSI score before surgery was $11.48 \pm 7.95$; this number decreased to $4.95 \pm 6.19$ after surgery $(P<0.001)$. The rate of positive RSI responses was 33\% before surgery and 9\% after surgery. Each variable that comprised the RSI improved significantly after surgery, except for difficulty with swallowing. Regarding the degree of RSI improvement after surgery, there were no significant differences between subgroups according to sex, age, body mass index, OSA severity, or surgical outcome.

Conclusion. LPR symptoms are prevalent in OSA patients. Treatment for OSA using multilevel surgery potentially reduces the symptoms of LPR.

Keywords. Obstructive Sleep Apnea; Laryngopharyngeal Reflux

\section{INTRODUCTION}

Obstructive sleep apnea (OSA) is caused by the collapse of the upper airway induced by negative pressure inspiration. It is characterized by repeated episodes of apnea and hypopnea during sleep, in spite of a normal respiratory drive. Approximately $2 \%-$ $4 \%$ of middle-aged people suffer from symptomatic OSA [1], a condition associated with significant cardiovascular morbidity and mortality [2]. Associations have been found between OSA

\footnotetext{
- Received January 12, 2017

Revised March 16, 2017

Accepted March 22, 2017

- Corresponding author: Hyo Yeol Kim

Department of Otorhinolaryngology-Head and Neck Surgery, Samsung Medical Center, Sungkyunkwan University School of Medicine, 81 Irwonro, Gangnam-gu, Seoul 06351, Korea

Tel: +82-2-3410-3579, Fax: +82-2-3410-3879

E-mail: siamkhy@gmail.com
}

and many systemic diseases, including hypertension, left ventricular dysfunction, arrhythmia, myocardial infarction, and stroke. Therefore, effective treatment, such as continuous positive airway pressure (CPAP) or multilevel surgery, should be applied promptly to reduce such cardiovascular, cerebrovascular, and neurocognitive complications.

Extraesophageal reflux is caused by the repeated retrograde overflow of gastric contents into the esophagus, and is divided into gastroesophageal reflux (GER) or laryngopharyngeal reflux (LPR) depending on the location of the injury. GER is defined as reflux that is limited to the esophagus, while LPR is defined as reflux that extends beyond the esophagus into the larynx and pharynx and is symptomatically characterized by laryngeal mucosal inflammation and damage. The incidence of LPR is high; nearly $10 \%$ of patients admitted to otolaryngology outpatient clinics complain of LPR [3]. LPR symptoms vary between individuals, ranging from hoarseness and vocal fatigue to throat

Copyright @ 2017 by Korean Society of Otorhinolaryngology-Head and Neck Surgery.

This is an open-access article distributed under the terms of the Creative Commons Attribution Non-Commercial License (http://creativecommons.org/licenses/by-nc/4.0)

which permits unrestricted non-commercial use, distribution, and reproduction in any medium, provided the original work is properly cited. 
clearing, postnasal drip, cough, dysphagia, and globus. Chronic untreated LPR can contribute to or cause several upper airway diseases, including laryngopharyngitis, laryngospasm, and subglottic stenosis, as well as benign and malignant diseases of the head and neck $[4,5]$.

Both OSA and LPR are common chronic diseases that frequently coexist. They share several risk factors, including obesity, male predominance, alcohol use, and age. The coexistence of OSA with LPR has been reported to have a prevalence of $20 \%-$ $67 \%[6,7]$. Several earlier studies explored a possible association between OSA and LPR $[7,8]$. Some studies evaluated the effect of antireflux therapy on OSA and reported improvements in the symptoms or parameters of OSA $[9,10]$. Other studies demonstrated the effect of CPAP treatment on reflux disease and showed significant improvements in reflux symptoms $[11,12]$. Although previous studies were unable to demonstrate a direct temporal or causal relationship between OSA and LPR, they did suggest a possible bidirectional causative relationship between OSA and LPR [13].

However, to date no studies have investigated the effect of surgical treatment for OSA on reflux disease. The aim of this study was to evaluate the change in laryngeal reflux symptoms after multilevel surgery for the treatment of OSA, based on patients' responses to the reflux symptom index (RSI) questionnaire.

\section{MATERIALS AND METHODS}

\section{Subjects}

This observational study (a retrospective chart review) consisted of patients who were diagnosed with OSA and who underwent multilevel surgery (uvulopalatopharyngoplasty, tonsillectomy, radiofrequency tongue base reduction, with or without nasal surgery) at a single tertiary medical center between April 2009 and September 2014. This study was approved by the Institutional Review Board of Samsung Medical Center (No. 201702119001). All operations were performed by a single surgeon (HYK). All patients were asked to complete an RSI questionnaire before and at least 6 months after surgery. Patients lost to

\section{H I}

- Laryngeal reflux symptoms are prevalent in patients with obstructive sleep apnea (OSA).

- The reflux symptom index (RSI) decreased significantly after multilevel surgery to treat OSA.

- The RSI-positivity rate also decreased significantly, from $33 \%$ to $9 \%$, after surgery.

- We did not identify any factor influencing any difference in the extent of improvement among subgroups. follow-up; who did not complete the follow-up questionnaire; who used antireflux medication (either before or after surgery); who had undergone previous antireflux surgery; or who had a history of systemic, major thoracic, or abdominal illness or surgery, were excluded. The age, height, weight, body mass index (BMI), and results of polysomnography (PSG) for each patient were obtained via a retrospective chart review in order to evaluate changes in the RSI.

\section{Polysomnography}

Complete overnight diagnostic PSG was performed using Alice3 (Healthdyne Technologies, Marietta, GA, USA) or Somnologica Studio (Embla Systems, Broomfield, CO, USA) PSG devices prior to surgery to monitor electroencephalogram (EEG; C3/ A2, C4/A1, O1/A2, and O2/A1), 4-channel electrooculogram, electromyogram (submental, intercostal, and anterior tibialis muscles), and electrocardiogram values; abdominal and thoracic movements (using piezoelectric bands); nasal-oral airflow; and oxygen saturation according to pulse oximetry [14]. Postoperative PSG was not routinely scheduled, being performed only on particular patients who required follow-up testing or who seemed to exhibit no improvement after surgery.

PSG was scored according to standard rules [15]. The apneahypopnea index (AHI), defined as the number of apnea and hypopnea events per hour of sleep, was the primary PSG outcome measure. A diagnosis of OSA was made for patients with an AHI value $>15$. OSA was classified in this study as mild (AHI, $5-15$ ), moderate (AHI, $>15$ and $\leq 30$ ), or severe (AHI, $>30$ ). Surgical success was defined as a postoperative AHI value $\leq 20$ with a $50 \%$ reduction in the AHI from baseline [16].

\section{Reflux symptom index}

All patients were asked to complete an RSI questionnaire both before surgery and at least 6 months afterwards. The RSI questionnaire is a widely used and approved, self-administered, nineitem questionnaire for the assessment of symptoms in LPR patients. The questionnaire consists of questions regarding hoarseness, throat clearing, postnasal drip, difficulty with swallowing, coughing, breathing difficulty, troublesome cough, lump sensation, and heartburn. The scale for each item ranges from 0 (no problem) to 5 (severe problem), with a maximum total score of 45 . A total score $>13$ indicates that the patient has reflux symptoms [17].

\section{Statistical analysis}

Differences in patient characteristics between those with reflux symptoms (RSI score $>13$ ) and those with no symptoms (RSI score $\leq 13$ ) were analyzed using the chi-squared test, Student $t$ test, or Mann-Whitney $U$-test, as appropriate. The Wilcoxon signed-rank test was used to compare RSI scores before and after surgery. We performed additional comparisons after stratifying the patients according to sex, age, BMI, OSA severity, and 
Table 1. Characteristics of patients with obstructive sleep apnea $(n=73)$

\begin{tabular}{lc}
\hline Characteristic & Value \\
\hline Sex & \\
$\quad$ Male & $62(85)$ \\
Female & $11(15)$ \\
Age $(\mathrm{yr})$ & $42.3 \pm 11.8$ \\
Body mass index $\left(\mathrm{kg} / \mathrm{m}^{2}\right)$ & $25.88 \pm 3.02$ \\
AHI & $27.74 \pm 19.75$ \\
Supine AHI & $42.74 \pm 25.14$ \\
Lateral AHI & $12.61 \pm 18.39$ \\
RSI & \\
$>13$ & $24(33)$ \\
$\leq 13$ & $49(67)$ \\
\hline
\end{tabular}

Values are presented as number (\%) or mean \pm standard deviation.

$\mathrm{AHI}$, apnea-hypopnea index; RSI, reflux symptom index.

surgical outcome. The Mann-Whitney $U$-test or a one-way analysis of variance using the Kruskal-Wallis test were used, as appropriate, to compare the degree of improvement between groups. All statistical analyses were performed with IBM SPSS ver. 23.0 (IBM Co., Armonk, NY, USA). A $P$-value less than 0.05 was considered to indicate statistical significance for all analyses.

\section{RESULTS}

\section{Subjects}

Ninety-two patients underwent multilevel surgery for OSA after complete overnight diagnostic polysomnography. Of these, 73 (62 males and 11 females) who completed RSI questionnaires were included in the present study. Eleven patients were excluded because they were either lost to follow-up or did not complete the questionnaires. An additional 8 patients were excluded because they used antireflux medication after surgery. The final study population thus consisted of 73 patients.

Table 1 lists patient demographic characteristics. Of the 73 patients, $62(85 \%)$ were male and $11(15 \%)$ were female. The patients ranged in age from 16 to 66 years, with a mean age of $42.3 \pm 11.8$ years. The mean BMI was $25.88 \pm 3.02 \mathrm{~kg} / \mathrm{m}^{2}$, with a range of $20.00-36.96 \mathrm{~kg} / \mathrm{m}^{2}$. All patients were diagnosed with OSA and had a mean AHI score of $27.74 \pm 19.75$. Of the 73 patients, 24 (33\%) had RSI scores $>13$ and were considered to have reflux. The remaining $49(67 \%)$ had RSI scores $\leq 13$ and were considered to not have reflux.

\section{Comparison of reflux symptoms before and after surgery}

Table 2 shows the RSI scores before and after multilevel surgery for OSA. The mean total RSI score was significantly decreased after surgery. The RSI positive rate was also significantly decreased from $33 \%$ to $9 \%$ after surgery. All individual variables in the RSI, with the exception of difficulty with swallowing
Table 2. Reflux symptom index scores before and after multilevel surgery to treat obstructive sleep apnea

\begin{tabular}{lccr}
\hline \multirow{2}{*}{ Variable } & \multicolumn{2}{c}{ Reflux symptom index } & \multirow{2}{*}{$P$-value ${ }^{\text {a) }}$} \\
\cline { 2 - 3 } & Before surgery & After surgery & \\
\hline 1. Hoarseness & $1.00 \pm 1.12$ & $0.34 \pm 0.72$ & $<0.001$ \\
2. Throat-clearing & $1.79 \pm 1.30$ & $0.77 \pm 1.21$ & $<0.001$ \\
3. Postnasal drip & $2.08 \pm 1.43$ & $0.25 \pm 0.73$ & $<0.001$ \\
4. Difficulty swallowing & $0.46 \pm 1.12$ & $0.25 \pm 0.73$ & 0.084 \\
5. Coughing & $0.53 \pm 0.86$ & $0.26 \pm 0.69$ & 0.017 \\
6. Breathing difficulty & $1.28 \pm 1.43$ & $0.49 \pm 1.09$ & $<0.001$ \\
7. Troublesome cough & $0.65 \pm 1.12$ & $0.22 \pm 0.56$ & 0.002 \\
8. Lump sensation & $1.62 \pm 1.50$ & $0.93 \pm 1.40$ & 0.002 \\
9. Heartburn & $1.84 \pm 1.49$ & $0.55 \pm 1.11$ & $<0.001$ \\
Total reflux symptom index & $11.48 \pm 7.95$ & $4.95 \pm 6.19$ & $<0.001$ \\
\hline
\end{tabular}

Values are presented as means \pm standard deviation.

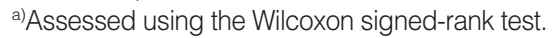

Table 3. Changes in reflux symptom index scores by reference to various factors

\begin{tabular}{lcc}
\hline Variable & Difference $^{\mathrm{a}}$ & $P$-value \\
\hline Sex & & 0.326 \\
$\quad$ Male & $5.98 \pm 7.73$ & \\
$\quad$ Female & $9.36 \pm 9.57$ & 0.840 \\
Age $(\mathrm{yr})$ & & \\
$<30$ & $6.40 \pm 8.15$ & \\
$30-49$ & $6.05 \pm 6.71$ & \\
$\geq 50$ & $6.00 \pm 8.26$ & \\
Body mass index & & \\
Normal & $6.71 \pm 7.99$ & \\
Overweight & $6.28 \pm 8.49$ & 0.770 \\
Obese & $7.00 \pm 5.79$ & \\
OSA severity & & \\
Mild & $7.05 \pm 6.92$ & \\
Moderate & $6.15 \pm 7.89$ & 0.352 \\
Severe & $6.91 \pm 9.03$ & \\
Surgical outcome & & \\
Successful & $6.61 \pm 7.35$ & \\
Unsuccessful & $5.23 \pm 9.72$ & \\
\hline
\end{tabular}

Values are presented as means \pm standard deviation.

RSI, reflux symptom index; OSA, obstructive sleep apnea.

a)The difference in the reflux symptom index score before and after surgery (preoperative total RSI score-postoperative total RSI score). ${ }^{\text {b) Assessed }}$ using the Kruskal-Wallis test (except for sex and surgical outcome, which were assessed with the aid of the Mann-Whitney U-test).

$(P=0.084)$, improved significantly after surgery $(P<0.05)$.

\section{Comparison of changes in reflux symptoms according to vari- ous factors}

Table 3 shows a comparison of the changes in the RSI following surgery after stratifying by sex, age, BMI, OSA severity, and surgical outcome. Each group showed a significant improvement in the mean RSI score after surgery $(P<0.05)$; however, there was no significant difference in the degree of improvement between the groups $(P>0.05)$. 


\section{DISCUSSION}

In this study, we compared laryngeal reflux symptoms based on patient responses to the RSI questionnaire before and after multilevel surgery for the treatment of OSA. The major finding of this study is that laryngeal reflux symptoms, which are more prevalent in OSA patients than in the general population, were improved significantly after multilevel surgery not only in terms of the total RSI score but also individual variables. To our knowledge, no previous studies have investigated the effect of surgical treatment for OSA on the symptoms of reflux. In this study, we directly compared laryngeal reflux symptoms before and after surgery.

Several studies have attempted to demonstrate a temporal relationship between reflux disease and OSA; however, the underlying pathophysiological mechanisms between OSA and reflux diseases, especially LPR, are not well understood [13]. A number of published studies that evaluated the association between OSA and reflux disease focused on GER rather than LPR, reporting increased negative intrathoracic pressure during obstructive events that could possibly overcome the barrier pressure provided by the lower esophageal sphincter [18]. Additionally, repetitive arousals terminating obstructive events could increase OSA patient susceptibility to GER $[19,20]$.

CPAP, the mainstay therapy for OSA, can reduce GER events and improve nocturnal GER symptoms in OSA patients. The mechanism by which CPAP reduces GER symptoms has been explained as follows: CPAP elevates the intrathoracic pressure by reducing airway obstruction and inspiratory effort and by the transmission of positive pressure to the intrathoracic airways. By increasing the intraesophageal pressure, the risk of GER should be reduced in patients with OSA. Other potential mechanisms include an increase in lower esophageal sphincter pressure by reflex mechanisms or direct mechanical compression of the midesophagus [12,21].

Unlike these previous studies, we focused instead on LPR and its relationship to OSA. LPR has been recognized not simply as the laryngopharyngeal manifestation of GER, but rather as a distinct entity within the spectrum of gastric material reflux syndromes, with characteristic double-pH-probe profiles and clinical symptoms $[22,23]$. While GER is primarily associated with the backflow of gastric contents into the esophagus due to lower esophageal sphincter dysfunction, with relative protection of the upper airway by the upper esophageal sphincter, LPR occurs with minimal esophageal reflux but upper esophageal sphincter malfunction, resulting in laryngeal mucosal injury and laryngopharyngeal symptoms [22-24].

In this vicious cycle between OSA and LPR, progressive increases in respiratory effort by an OSA patient can produce greater negative intrathoracic pressure. This negative intrathoracic pressure promotes the reflux of gastric contents into the esophagus, larynx, and pharynx. Over time, LPR-induced in- flammation contributes to OSA progression in two ways: (1) inflammation-mediated thickening and hypertrophy of tissues that narrow the upper airway directly and (2) chronic tissue damage and sensory impairment that cause dysfunction in reflexes important in maintaining upper airway patency during sleep [13].

The laryngeal epithelium is relatively more susceptible to the hazardous effect of gastric contents than the esophageal mucosa. Even a few episodes of reflux can cause LPR disease, which can lead to mucosal injury and inflammation $[5,25]$. Increased sensitivity of the laryngopharyngeal mucosa (compared to the esophageal mucosa) to reflux-induced inflammation presumably contributes to inflammatory changes in the upper airway mucosa and OSA progression [13].

OSA not only contributes to inflammatory changes in the laryngeal mucosa through this cycle of harm, but also encourages oropharyngeal tissue inflammation through its own pathophysiologic mechanism. Previous studies have proposed that these inflammatory changes might be due in part to mechanical trauma related to vibration-induced injury during snoring or forceful suction collapse and traction during apnea and hypopnea events. Another mechanism that might exert proinflammatory influences is repeated hypoxia-reoxygenation, which is characteristic of OSA and leads to the generation of free radicals and increased oxidative stress [24,26,27].

In our study, we presumed that multilevel surgery could reduce snoring-induced mechanical trauma arising from vibration and forceful suction collapse and traction during apnea events, and, as a result, prevent oropharyngeal tissue inflammation and improve reflux symptoms via improved upper airway patency. Multilevel surgery also reduces respiratory effort, reducing increases in intrathoracic pressure and the reflux of gastric contents and consequently improving LPR-induced inflammation and symptoms related to LPR.

In clinical settings, patients often complain of subjective discomfort after multilevel surgery, including pharyngeal pain, difficulty with swallowing, sensation of a foreign body in the throat, and globus. A considerable number of these symptoms are similar to those listed in the RSI questionnaire. Another primary intention of this study was to compare RSI scores before and after multilevel surgery in order to verify such postoperative complaints. Contrary to expectations, most of these symptoms improved significantly after surgery.

When we compared the pre- and post-surgery changes in RSI score according to sex, age, BMI, OSA severity, and surgical outcome, each group experienced a significant decrease in the RSI score, but the differences in terms of the degree of change were not significant between the groups. Regarding surgical outcome, successful treatment was defined as a postoperative AHI value $\leq 20$ with a $50 \%$ reduction in the AHI from baseline [16]. The surgical outcome was entirely determined by the AHI value, and other factors were not considered. There are several possible reasons why the surgical outcome had no influence on the degree 
of RSI improvement when comparing patients with successful surgeries to those whose surgeries were not successful. First of all, because not all patients underwent follow-up PSG, those patients analyzed for surgical success were unable to represent the entire group of patients. Patients who required follow-up testing or who seemed to show no improvement after surgery were subjected to follow-up PSG. Of all 73 patients, only 31 underwent postoperative follow-up PSG. Of these, 18 exhibited surgical success; the mean AHI improved from 23.48 to 6.97. Reflux symptom scores also improved, from 13.83 to 7.22 . Surgery was not successful in the remaining 13 patients; the mean AHIs before and after surgery were 31.33 and 28.06 , respectively. However, these patients did exhibit significant improvements in reflux symptoms; the scores before and after surgery were 14.08 and 8.85, respectively. Further studies featuring follow-up PSG may explain the observed differences in surgical outcomes. Second, use of the AHI as the sole measure in the assessment of surgical outcome is problematic [28,29]. Previous studies emphasized that the assessment of the surgical outcome for OSA should neither be limited to the AHI as an outcome, nor should it be the only outcome emphasized [29]. Third, regardless of improvement in the AHI, patients could have experienced an improvement in snoring, which might allow them to avoid mechanical trauma related to vibration-induced injury during snoring and eliminate inflammation in oropharyngeal tissue and laryngeal mucosa, which can improve reflux symptoms. In addition, patients who had a mechanical obstruction such as palatine tonsillar hypertrophy could have experienced improvement simply by resolving the mechanical obstruction with surgery.

There were some limitations to our study. First, we assessed LPR using only a subjective questionnaire based on patient selfreporting, rather than a more objective measure such as 24-hour double-probe $\mathrm{pH}$ monitoring or endoscopic examination. The primary intention of this study was to assess changes in reflux symptoms after multilevel surgery because OSA patients often complain of symptoms that are similar to those included in the RSI questionnaire after multilevel surgery. Contrary to our expectations, the total RSI score and its individual variables improved significantly after multilevel surgery. Nevertheless, our study would be strengthened by the use of an objective test in addition to these subjective measures. Second, we did not analyze anatomical variations that could affect the surgical outcome and changes in reflux symptoms. Multilevel surgery is a combination of various operations rather than a single operation. Therefore, surgical outcomes may differ by the site of obstruction and the surgery performed. However, all of our present exhibited (principally) retropalatal obstructions, and required uvulopalatopharyngoplasties. Tonsillectomy and radiofrequency tongue base reduction were also performed in all cases. The nasal surgeries were commonly performed (50 cases of turbinoplasty, 43 cases of septoplasty, and 7 cases of endoscopic sinus surgery), but made no fundamental difference. Additional stud- ies including more cases are necessary to explore whether anatomical factors exert potentially causal effects. Third, this study was not performed in a blinded fashion and did not include a control group. However, the aim of this study was to evaluate the surgical results, which is difficult to conduct in a randomized controlled fashion. Had we been able to, we could have compared matched patients based on CPAP treatment.

In conclusion, OSA treatment via multilevel surgery potentially reduces laryngeal reflux symptoms. Additional studies using objective measures to evaluate the effects of different anatomical features are needed to clarify these results.

\section{CONFLICT OF INTEREST}

No potential conflict of interest relevant to this article was reported.

\section{REFERENCES}

1. Young T, Palta M, Dempsey J, Skatrud J,Weber S, Badr S. The occurrence of sleep-disordered breathing among middle-aged adults. N Engl J Med. 1993 Apr;328(17):1230-5.

2. Jean-Louis G, Zizi F, Clark LT, Brown CD, McFarlane SI. Obstructive sleep apnea and cardiovascular disease: role of the metabolic syndrome and its components. J Clin Sleep Med. 2008 Jun;4(3):261-72.

3. Koufman JA. The otolaryngologic manifestations of gastroesophageal reflux disease (GERD): a clinical investigation of 225 patients using ambulatory 24-hour $\mathrm{pH}$ monitoring and an experimental investigation of the role of acid and pepsin in the development of laryngeal injury. Laryngoscope. 1991 Apr;101(4 Pt 2 Suppl 53):1-78.

4. Groome M, Cotton JP, Borland M, McLeod S, Johnston DA, Dillon JF. Prevalence of laryngopharyngeal reflux in a population with gastroesophageal reflux. Laryngoscope. 2007 Aug;117(8):1424-8.

5. Koufman JA, Aviv JE, Casiano RR, Shaw GY. Laryngopharyngeal reflux: position statement of the committee on speech, voice, and swallowing disorders of the American Academy of OtolaryngologyHead and Neck Surgery. Otolaryngol Head Neck Surg. 2002 Jul;127 (1):32-5.

6. Zanation AM, Senior BA.The relationship between extraesophageal reflux (EER) and obstructive sleep apnea (OSA). Sleep Med Rev. 2005 Dec;9(6):453-8.

7. Wise SK, Wise JC, DelGaudio JM. Gastroesophageal reflux and laryngopharyngeal reflux in patients with sleep-disordered breathing. Otolaryngol Head Neck Surg. 2006 Aug;135(2):253-7.

8. Ing AJ, Ngu MC, Breslin AB. Obstructive sleep apnea and gastroesophageal reflux. Am J Med. 2000 Mar;108 Suppl 4a:120S-125S.

9. Senior BA, Khan M, Schwimmer C, Rosenthal L, Benninger M. Gastroesophageal reflux and obstructive sleep apnea. Laryngoscope. 2001 Dec;111(12):2144-6.

10. Steward DL. Pantoprazole for sleepiness associated with acid reflux and obstructive sleep disordered breathing. Laryngoscope. 2004 Sep; 114(9):1525-8.

11. Green BT, Broughton WA, O'Connor JB. Marked improvement in nocturnal gastroesophageal reflux in a large cohort of patients with obstructive sleep apnea treated with continuous positive airway pressure. Arch Intern Med. 2003 Jan;163(1):41-5.

12. Tawk M, Goodrich S, Kinasewitz G, Orr W. The effect of 1 week of 
continuous positive airway pressure treatment in obstructive sleep apnea patients with concomitant gastroesophageal reflux. Chest. 2006 Oct;130(4):1003-8.

13. Eskiizmir G, Kezirian E. Is there a vicious cycle between obstructive sleep apnea and laryngopharyngeal reflux disease? Med Hypotheses. 2009 Nov;73(5):706-8.

14. Kim HY, Jeong JI, Dhong HJ, Sohn JH, Hong SD, Kim JH, et al. Nasal obstruction and palate-tongue position on sleep-disordered breathing. Clin Exp Otorhinolaryngol. 2013 Dec;6(4):226-30.

15. Berry RB, Budhiraja R, Gottlieb DJ, Gozal D, Iber C, Kapur VK, et al. Rules for scoring respiratory events in sleep: update of the 2007 AASM manual for the scoring of sleep and associated events: deliberations of the Sleep Apnea Definitions Task Force of the American Academy of Sleep Medicine. J Clin Sleep Med. 2012 Oct;8(5):597619.

16. Elshaug AG, Moss JR, Southcott AM, Hiller JE. Redefining success in airway surgery for obstructive sleep apnea: a meta analysis and synthesis of the evidence. Sleep. 2007 Apr;30(4):461-7.

17. Belafsky PC, Postma GN, Koufman JA.Validity and reliability of the reflux symptom index (RSI). JVoice. 2002 Jun;16(2):274-7.

18. GislasonT, Janson C,Vermeire P, Plaschke P, Bjornsson E, Gislason D, et al. Respiratory symptoms and nocturnal gastroesophageal reflux: a population-based study of young adults in three European countries. Chest. 2002 Jan;121(1):158-63.

19. Penzel T, Becker HF, Brandenburg U, Labunski T, Pankow W, Peter $\mathrm{JH}$. Arousal in patients with gastro-oesophageal reflux and sleep apnoea. Eur Respir J. 1999 Dec;14(6):1266-70.

20. Shepherd K, Hillman D, Holloway R, Eastwood P. Mechanisms of nocturnal gastroesophageal reflux events in obstructive sleep apnea. Sleep Breath. 2011 Sep;15(3):561-70.
21. Fournier MR, Kerr PD, Shoenut JP, Yaffe CS. Effect of nasal continuous positive airway pressure on esophageal function. J Otolaryngol. 1999 Jun;28(3):142-4.

22. Koufman JA. Laryngopharyngeal reflux is different from classic gastroesophageal reflux disease. Ear NoseThroat J. 2002 Sep;81(9 Suppl 2):7-9.

23. Postma GN, Tomek MS, Belafsky PC, Koufman JA. Esophageal motor function in laryngopharyngeal reflux is superior to that in classic gastroesophageal reflux disease. Ann Otol Rhinol Laryngol. 2001 Dec;110(12):1114-6.

24. Payne RJ, Kost KM, Frenkiel S, Zeitouni AG, Sejean G, Sweet RC, et al. Laryngeal inflammation assessed using the reflux finding score in obstructive sleep apnea. Otolaryngol Head Neck Surg. 2006 May; 134(5):836-42.

25. Lipan MJ, Reidenberg JS, Laitman JT. Anatomy of reflux: a growing health problem affecting structures of the head and neck. Anat Rec B New Anat. 2006 Nov;289(6):261-70.

26. Boyd JH, Petrof BJ, Hamid Q, Fraser R, Kimoff RJ. Upper airway muscle inflammation and denervation changes in obstructive sleep apnea.Am J Respir Crit Care Med. 2004 Sep;170(5):541-6.

27. Bergeron C, Kimoff J, Hamid Q. Obstructive sleep apnea syndrome and inflammation. J Allergy Clin Immunol. 2005 Dec;116(6):13936.

28. Hobson JC, Robinson S, Antic NA, McEvoy RD, Windler S, MacKay $\mathrm{S}$, et al. What is "success" following surgery for obstructive sleep apnea? The effect of different polysomnographic scoring systems. Laryngoscope. 2012 Aug;122(8):1878-81.

29. Tam S, Woodson BT, Rotenberg B. Outcome measurements in obstructive sleep apnea: beyond the apnea-hypopnea index. Laryngoscope. 2014 Jan;124(1):337-43. 